\title{
Evaluating Visibility of Age Spot and Freckle Based on Simulated Spectral Reflectance of Skin
}

\author{
Misa Hirose $^{1}$, Saori Toyota ${ }^{1}$, Yuri Tatsuzawa ${ }^{2}$, and Norimichi Tsumura ${ }^{1}$ \\ 1 Graduate School of Advanced Integration Science, Chiba University, Chiba, Japan \\ 2 Department of Informatics and Imaging Systems, Chiba University, Chiba, Japan
}

\begin{abstract}
In this research, we evaluated the visibility of age spot and freckle by changing volume of pigmentations and the spatial distribution of age spot and freckle based on the spectral reflectance of skin. The spectral reflectance is simulated by using Monte Carlo simulation of light transport in multi-layered tissue. Three type of spatial distributions of age spot and freckle are generated based on the simulated spectral reflectance. We performed subjective evaluation for the visibility of the simulated age spot and freckle patterns, and found age spot and freckle become less noticeable as the increase of blood volume.
\end{abstract}

Keywords: age spot, melanin volume, blood volume, Monte Carlo simulation, spectral reflectance.

\section{Introduction}

Age spot and freckle in the skin is caused by UV irradiation and aging. Since human face and skin receive a lot of attention in the human body, appearances of age and the health condition are caused by these pigmentations. Therefore, women are interested in cosmetics to reduce the visibility of these pigmentations, and various studies have been performed in cosmetology and medical science for this reduction of visibility. Skin color is determined by the pigments such as melanin in epidermis and hemoglobin in dermis. It is known that melanin is excessively generated in epidermis at the region of age spot and freckle, and the region is observed as darker region compared to the region of normal skin.

Lihong Wang and Steven L. Jacques produced a standard C-code for Monte Carlo simulation of light transport in multi-layered tissue (MCML) [1. MCML analyzes photon propagation in multi-layered tissue and estimates spectral reflectance. Okamoto et al. produced Monte Carlo simulation of light reflection from cosmetic powder particles near the human skin surface [2], and calculated color difference of pigmented area and skin applied the cosmetic powder. However, the relationship is not well understood between the visibility and amount of pigmentations.

In this research, therefore, we evaluate the visibility of age spot and freckle by changing melanin and blood volume and the spatial distribution of age spot and freckle based on the spectral reflectance of skin. The spectral reflectance is simulated by using MCML with changing the melanin and blood volume.

A. Elmoataz et al. (Eds.): ICISP 2014, LNCS 8509, pp. 9-17, 2014.

(C) Springer International Publishing Switzerland 2014 
Three type of spatial distributions of age spot and freckle are generated based on the simulated spectral reflectance. We perform subjective evaluation for the visibility of the simulated age spot and freckle patterns.

\section{Monte Carlo Simulation for Photon Migration}

Lihong Wang and Steven L. Jacques produced a standard C-code for Monte Carlo simulation of light transport in multi-layered tissue (MCML). As shown in Fig 1, MCML is constituted by following operations for photons. Our research utilizes the MCML to simulate the spectral reflectance of normal skin, age spot and freckle.

First, a photon is launched into the tissue. Propagation distance of photons equals to $\Delta s=-\operatorname{In}(\xi) /\left(\mu_{a}+\mu_{s}\right)$ where $\xi$ is a uniform random numbers between $\mathrm{O}$ and $1, \mu_{a}$ and $\mu_{s}$ are absorption and scattering coefficients respectively. The position of the photon is specified by the Cartesian coordinate $(x, y, z)$. The direction of photon movement is described by the directional cosines $\left(\mu_{x}, \mu_{y}, \mu_{z}\right)$. These are corresponding to the $x, y$ and $z$ axes, respectively. The photon position and directional cosines are initialized to $(0,0,0),(0,0,1)$ respectively. The new photon position $\left(x^{\prime}, y^{\prime}, z^{\prime}\right)$ are calculated for a photon at $(x, y, z)$ to the direction $\left(\mu_{x}, \mu_{y}, \mu_{z}\right)$ as follows.

$$
x^{\prime}=x+\mu_{x} \Delta s, y^{\prime}=y+\mu_{y} \Delta s, z^{\prime}=z+\mu_{z} \Delta s .
$$

Each photon packet is initially assigned a weight, W. If photon hit the tissue surface, specular reflectance $R_{s p}$ occur. Then the photon weight is decremented by specular reflectance.

The probability that the photon will be internally reflected is determined by the Fresnel reflection coefficient when the photon propagated across a boundary into a region with a different index of refraction. When photon is propagated into tissue, photon weight is absorbed by tissue. Attenuation of the photon weight due to the absorption is computed by absorption and scattering coefficient. After the photon weight is absorbed, photon is scattered. The direction in which photon is scattered is computed by phase function using the uniform random numbers.

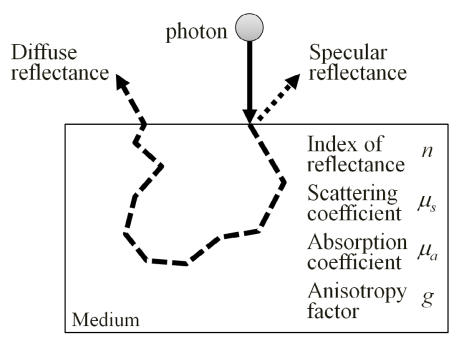

Fig. 1. The movement of photon through a medium calculated by Monte Carlo simulation 


\section{Four-Layered Model for Human Skin}

In order to analyze the spectral reflectance based on MCML, we prepared fourlayered skin model composed of stratum corneum, epidermis, papillary dermis and reticular dermis as shown in Fig 2 [3. We set the thickness $t$, index of refraction $n$, scattering coefficient $\mu_{s}$, absorption coefficient $\mu_{a}$ and anisotropy factor $g$ for each layer. The scattering coefficient and the anisotropy factor 4] are shown in Fig $3(\mathrm{a})$.

The skin color is denoted by eumelanin and pheomelanin in epidermis, oxyhemoglobin, deoxyhemoglobin and bilirubin in papillary and reticular dermis, and $\beta$-carotene in all layer. In this research, we consider these 6 kinds of pigments. The absorption coefficient of eumelanin $\mu_{a . e u}$, pheomelanin $\mu_{\text {a.pheo }}$ [5] and baseline skin $\mu_{a . b a s e}$ such as organells, cell membranes and fibrils [6] shown in Fig $3(\mathrm{~b})$ are approximated as follows.

$$
\begin{aligned}
\mu_{\text {a.eu }} & =6.6 \times 10^{11} \times \lambda^{-3.33} \\
\mu_{\text {a.pheo }} & =2.9 \times 10^{15} \times \lambda^{-4.75} \\
\mu_{\text {a.base }} & =7.84 \times 10^{8} \times \lambda^{-3.255},
\end{aligned}
$$

where $\lambda$ is the wavelength as measured in nanometer. When molar extinction coefficients for oxyhemoglobin, deoxyhemoglobin, $\beta$-caroten and bilirubin [7] shown in Fig 3(d), Fig 3(c) are denoted by $\varepsilon_{o h b}, \varepsilon_{d o h}, \varepsilon_{c a r}$ and $\varepsilon_{b i l}$ respectively, the absorption coefficients for each pigment are calculated as follows.

$$
\begin{gathered}
\mu_{a . o h b}=2.303 \times \frac{\varepsilon_{o h b}}{66500} c_{h b} \\
\mu_{a . c a r}=2.303 \times \frac{\varepsilon_{c a r}}{537} c_{c a r} \\
\mu_{a . b i l}=2.303 \times \frac{\varepsilon_{b i l}}{585} c_{b i l},
\end{gathered}
$$

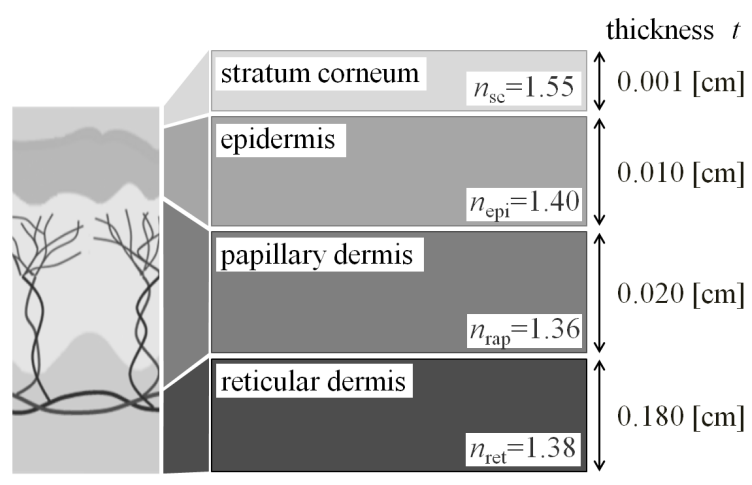

Fig. 2. Four-layered skin model 
where $\mu_{a . o h b}, \mu_{a . d h b}, \mu_{a . c a r}$ and $\mu_{a . b i l}$ are the absorption coefficient for each pigment. The values of 537, 585 and 66500 are molecular weight of each absorption, and $c_{c a r}, c_{b i l}$ and $c_{h b}$ are the concentration of $\beta$-carotene, bilirubin and hemoglobin respectively. The absorption coefficient of deoxyhemoglobin $\mu_{a . d h b}$ is computed by replacing $\varepsilon_{o h b}$ for $\varepsilon_{d h b}$. The concentration of hemoglobin $c_{h b}$ is typically $150[\mathrm{~g} / \mathrm{L}]$.

The absorption coefficient for each layer is calculated by the absorption coefficient and the volume fraction of pigments. The absorption coefficient of stratum corneum, epidermis denoted by $\mu_{a . s c}, \mu_{a . e p i}$ and the absorption coefficient of papillary and reticular dermis denoted by $\mu_{a \text {.der }}$ are given by

$$
\begin{aligned}
\mu_{a . s c} & =\mu_{a . b a s e}+\mu_{a . c s} \\
\mu_{a . e p i} & =\left(\mu_{a . e u} M_{e u}+\mu_{a . p h e o} M_{\text {pheo }}\right) M+\left(\mu_{a . b a s e}+\mu_{a . c e}\right)(1-M) \\
\mu_{a . d e r} & =\left(\mu_{a . o h b} S+\mu_{a . d h b}(1-S)+\mu_{a . c d}+\mu_{a . b i l}\right) B+\mu_{a . b a s e}(1-B),
\end{aligned}
$$

where $\mu_{a . c s}, \mu_{a . c e}$ and $\mu_{a . c d}$ are the absorption coefficient of $\beta$-carotene in stratum corneum, epidermis and dermis respectively, $M$ is the volume fraction of melanosomes in epidermis, $M_{e u}$ and $M_{p h e o}$ are the volume fraction of eumelanin and pheomelanin in melanosomes, $B$ is the volume fraction of whole blood in dermis, and $\mathrm{S}$ is the oxygen saturation.

\section{Generating Spatial Distribution of Pigmentations from Simulated Spectral Reflectance}

\subsection{The Concentration of Pigments for Skin, Age Spot and Freckle}

We determine the concentration of pigments for normal skin, age spot and freckle to generate spatial distribution of these pigmentation from spectral reflectance computed by MCML. The concentration of pigments for normal skin is determined to generate the average of measured spectral reflectance for 59 Japanese women. Then, the concentration of $\beta$-carotene is defined $22.2 \times 10^{-4}[\mathrm{~g} / \mathrm{L}]$ in stratum corneum and epidermis, $7.00 \times 10^{-4}[\mathrm{~g} / \mathrm{L}]$ in papillary dermis, $7.75 \times 10^{-4}$ $[\mathrm{g} / \mathrm{L}]$ in reticular dermis $[8$. The concentration of bilirubin is defined as a normal value of $7.00 \times 10^{-3}[\mathrm{~g} / \mathrm{L}]$. We define oxygen saturation as $75 \%$. Therefore, we estimate the volume fraction of melanosomes $M$, eumelanin $M_{e u}$, pheomelanin $M_{\text {pheo }}$ and whole blood $B$ from the average spectral reflectance. The measured spectral reflectance and the result of simulation by MCML are shown in Fig, 4, where we set $M=3.0 \%, M_{e u}=10.0 \%, M_{\text {pheo }}=90.0 \%$ and $B=3.0 \%$. The color difference $\Delta E$ in the CIELAB color space between the measurement and simulation was 0.923 . It is generally consider that we cannot perceive the difference between two colors if the value of $\Delta E$ is less than from 1 to $3[9$. Thus, we can conclude that the result of simulation is good performance for generating average of measured spectral reflectance. 


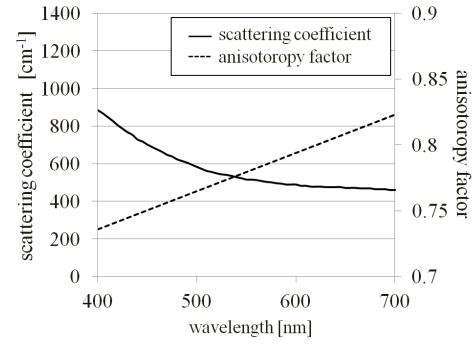

(a)

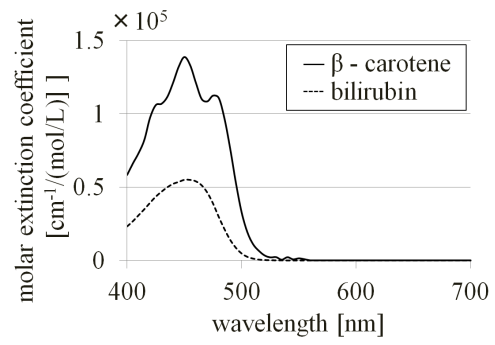

(c)

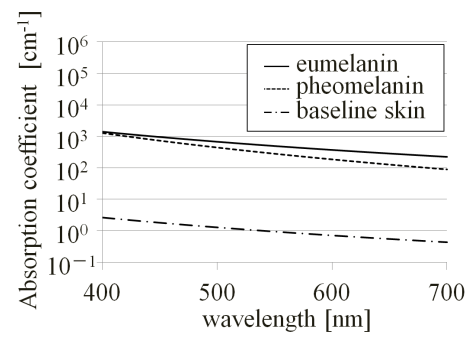

(b)

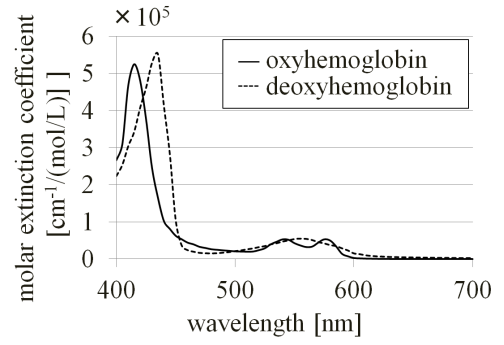

(d)

Fig. 3. (a) is scattering coefficient and anisotropy factor, (b) is absorption coefficient of eumelanin, pheomelanin and baseline skin, (c) is molar extinction coefficients of $\beta$-caroten and bilirubin, (d) is molar extinction coefficients for oxyhemoglobin and deoxyhemoglobin

We set that the volume fraction of melanosomes is more than $3.0 \%$ for age spot and freckle, because age spot and freckle are the regions where melanin is excessively generated in epidermis. Therefore, we set $M=4.0,5.0,6.0 \%$ in this research. For computing spectral reflectance by the change of blood volume, we increase the blood volume for skin and age spot from $3.0 \%$ to $20.0 \%$.

\subsection{Generating Spatial Distribution of Pigmentations}

We generate RGB image of age spot from spectral reflectance computed by MCML. First, we convert the spectral reflectance to XYZ color system as follows.

$$
\begin{aligned}
X & =k \int_{400}^{700} R(\lambda) P(\lambda) \bar{x}(\lambda) d \lambda \\
Y & =k \int_{400}^{700} R(\lambda) P(\lambda) \bar{y}(\lambda) d \lambda \\
Z & =k \int_{400}^{700} R(\lambda) P(\lambda) \bar{z}(\lambda) d \lambda \\
k & =1 / \int_{400}^{700} P(\lambda) \bar{y}(\lambda) d \lambda,
\end{aligned}
$$




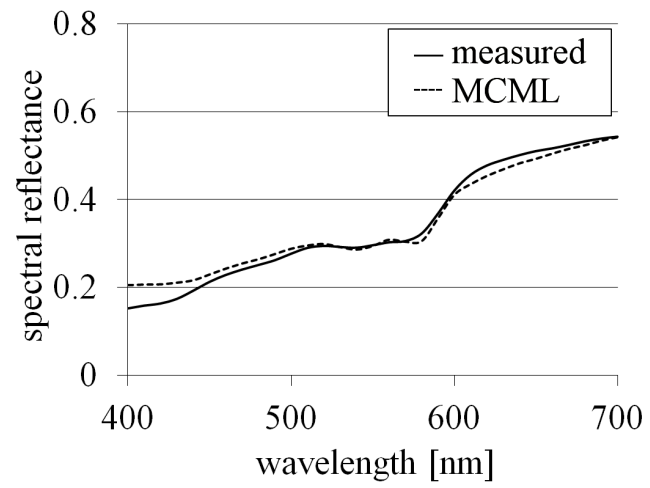

Fig. 4. Measured spectral reflectance and the result of simulation by MCML

where $R(\lambda)$ is spectral reflectance, $P(\lambda)$ is spectral distribution of the light source and $\bar{x}(\lambda), \bar{y}(\lambda), \bar{z}(\lambda)$ are color-matching function with a $2^{\circ}$ view as the observation condition [10]. The spectral distribution of the light source $P(\lambda)$ is 1.0 at all wavelength in this research. Next, we convert these to RGB as follows.

$$
\left(\begin{array}{l}
R \\
G \\
B
\end{array}\right)=\left(\begin{array}{rrr}
2.3655 & -0.8971 & -0.4683 \\
-0.5151 & 1.4264 & 0.0887 \\
0.0052 & -0.0144 & 1.0089
\end{array}\right)\left(\begin{array}{l}
X \\
Y \\
Z
\end{array}\right) .
$$

We perform gamma correction with $\gamma=2.4$ to be close to the color of actual skin. As shown in Fig 5 , we generate 3 kinds of spatial distribution of age spot because the actual age spot and freckle have various spatial distribution and sizes. The size of images is $500 \times 500$ (250,000 pixel), and age spot and freckle is 14,400 pixel. We apply a gaussian filter to blur the boundary of skin and age spot. The gaussian filter is defined by the kernel size and sigma $\sigma$. We set the kernel size $10 \times 10$ and $\sigma=10$.

Without split

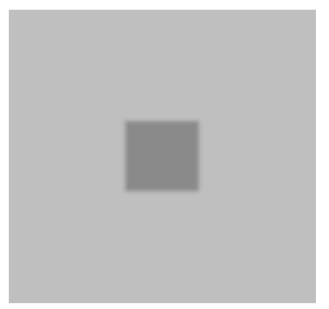

9 - split

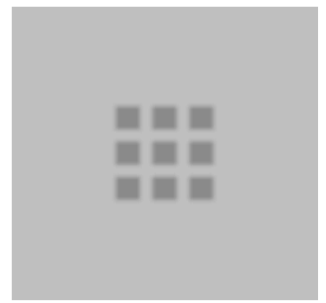

Random

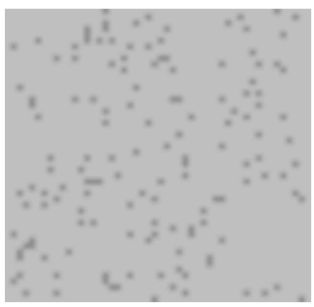

Fig. 5. The 3 kinds of distribution of age spot and freckle 


\section{Evaluating the Visibility of Age Spot and Freckle}

\subsection{Subjective Evaluation Method}

Figure 6(a) shows the experimental room. The display is 19 inch LCD and the viewing distance was approximately $87 \mathrm{~cm}$ that corresponded with three times the height of the display. In the subjective evaluation experiment, we used Thurstone's paired comparisons for evaluating the appearance of age spot and freckle by the change of blood volume. As shown in Fig 6(b), two images are displayed side-by-side at random on display. Observers select a image that age spot and freckle is less noticeable among two images. The number of observers is 12 .

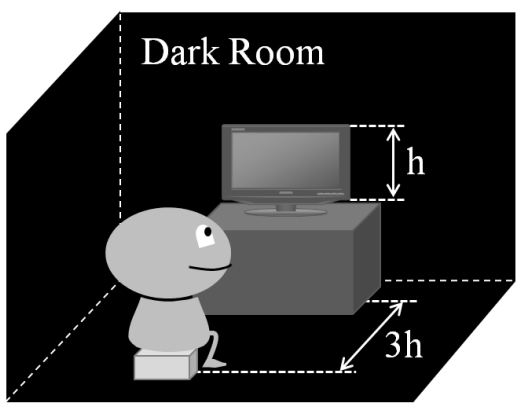

(a) Experimental room

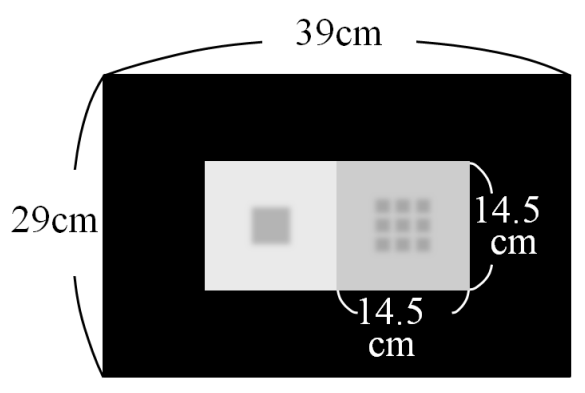

(b) Ratio of display to images

Fig. 6. Experimental set up

\subsection{Result and Discussion}

Figure 7 shows 27 images of age spot and freckle generated from spectral reflectance computed by MCML. The melanin volume of age spot and freckle increases from 4.0 to $6.0 \%$, and the blood volume in age spot and freckle and skin are $3.0,10.0$ and $20.0 \%$. There are 3 kinds of spatial distribution of age spot and freckle, that is without split, 9-split and random.

Figure 8 shows the result of subjective evaluation. The horizontal axis indicates the image numbers corresponding to numbers in Fig:7 The vertical axis indicate the subjectively evaluated value. This value becomes larger as the age spot and freckle become less noticeable. From the results, we can see that age spot and freckle become less noticeable as the increases of blood volume regardless of the distribution and melanin volume of age spot and freckle. We can also see that the visibility of age spot and freckle is changed depending on the spatial distribution of pigmentation and volume of melanin. 


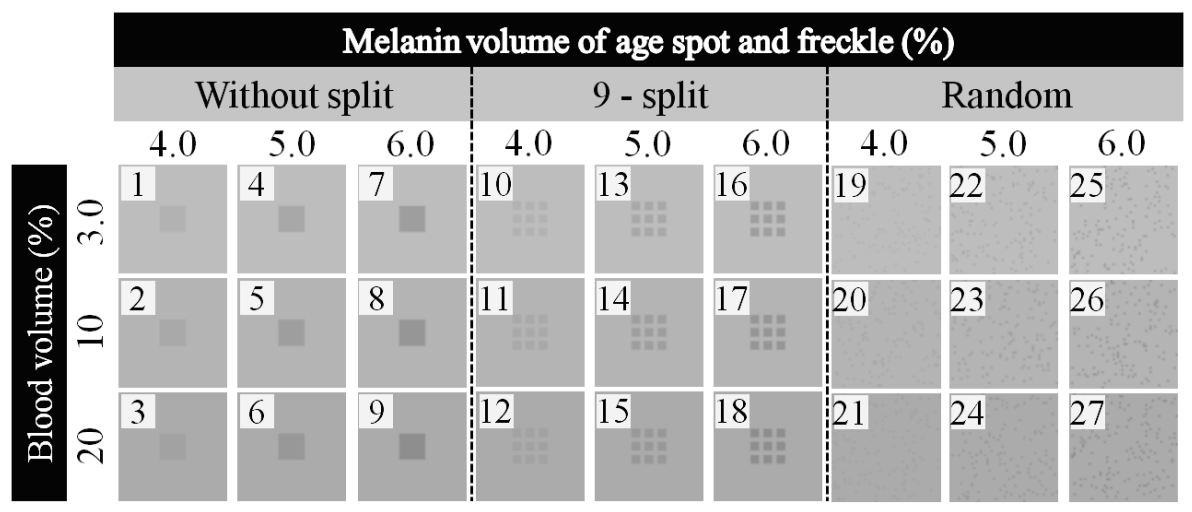

Fig. 7. Generated images of age spot and freckle

Image number

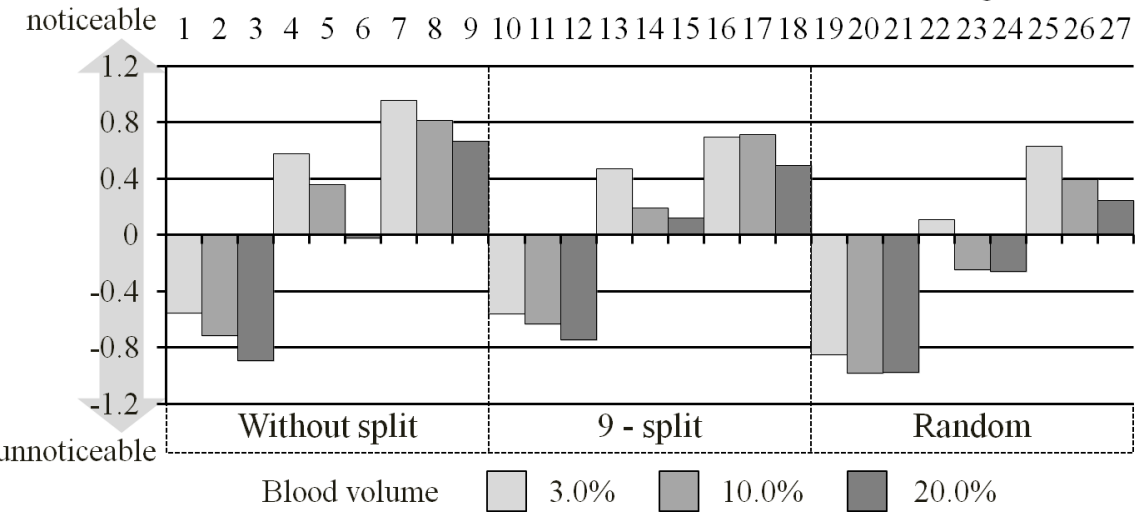

Fig. 8. The result of subjective evaluation

\section{Conclusion}

We generated the spatial distribution of age spot and freckle from spectral reflectance computed by MCML, and evaluated the visibility of pigmentation by the changing of blood volume for three type of spatial distributions. As a result of the subjective evaluation, we found that age spot and freckle become less noticeable as the increase of blood volume and the visibility is changed depending on the spatial distribution of pigmentation and volume of melanin.

The actual age spot and freckle are distributed on real human face, and the color of normal skin, age spot and freckle are not uniform. In the future work, we must investigate the effect of face shape and the color unevenness of skin. 
Acknowledgment. This work was supported by JSPS KAKENHI Grant Number 25135707 .

\section{References}

1. Wang, L., Jacques, S.L.: Monte Carlo Modeling of Light Transport in Multi-layered Tissues in Standard C. University of Texas M. D. Anderson Cancer Center (1992)

2. Okamoto, T., Kumagawa, T., Motoda, M., Igarashi, T., Nakao, K.: Monte Carlo simulation of light reflection from cosmetic powder particles near the human skin surface. Journal of Biomedical Optics 18(6), 061232, 1-11 (2013)

3. Krishnaswamy, A., Baranosk, G.V.G.: A Biophysically-Based Spectral Model of Light Interaction with Human Skin. EUROGRAPHICS 23(3), 331-340 (2004)

4. Tsumura, N., Kawabuchi, M., Haneishi, H., Miyake, Y.: Mapping Pigmentation in Human Skin from a Multi-Channel Visible Spectrum Image by Inverse Optical Scattering Technique. Journal of Imaging Science and Technology 45(5), 444-450 (2000)

5. Donner, C., Jensen, H.W.: A Spectral BSSRDF for Shading Human Skin. EUROGRAPHICS, 409-417 (2006)

6. Jacques, S.L.: Skin Optics, Organ Medical Center News (1998), http://omlc.ogi.edu/news/jan98/skinoptics.html

7. Oregon Medical Laser Center, http://omlc.ogi.edu/

8. Anders Vahlquist, M.D., et al.: Vitamin A in Human Skin: II Concentrations of Carotene, Retinol and Dehydroretinol in Various Compornents of Normal Skin. Journal of Investigative Dermatology 79(2), 94-97 (1982)

9. Hunt, R.W.G.: Measuring Color, Fountain, London (1998)

10. Colour Matching Functions, http://cvrl.ioo.ucl.ac.uk/cmfs.htm 